\title{
Getting out of crises: Environmental, social-ecological and evolutionary research needed to avoid future risks of pandemics
}

Delphine Destoumieux-Garzón*a, Franziska Matthies-Wiesler*b, Nicolas Biernec, Aurélie Binotd, Jérôme Boissiera, Anaïs Devouge ${ }^{\mathrm{e}}$, Jeanne Garric ${ }^{\mathrm{f}}$, Kim Gruetzmacherg, Christoph Grunaua, JeanFrançois Guégand,h, Sylvie Hurtrez-Boussès ${ }^{\mathrm{h}, \mathrm{i}}$, Anke Hussi, Serge Morandk, Clare Palmerl, Denis Sarigiannis ${ }^{\mathrm{m}, \mathrm{n}}$, Roel Vermeuleni, Robert Baroukie.

a IHPE, Univ Montpellier, CNRS, IFREMER, Univ Perpignan Via Domitia, Montpellier, France

${ }^{b}$ Institute of Epidemiology, Helmholtz Zentrum München, German Research Center for Environmental Health, Germany

${ }^{c}$ ISEM, Univ Montpellier, CNRS, EPHE, IRD, Montpellier, France

${ }^{d}$ Animals, health, Territories, Risks and Ecosystem (ASTRE), University of Montpellier, Agricultural research for Development (CIRAD), National Research Institute for Agriculture, Food and the Environment (INRAE), Montpellier, France

e INSERM, Université de Paris, France

fInstitut national de recherche pour l'agriculture, l'alimentation et l'environnement (INRAE), UR Riverly, F69625 Villeurbanne, France

g Museum für Naturkunde - Leibniz Institute for Evolution and Biodiversity Science, Berlin Germany

${ }^{h}$ MIVEGEC, Univ Montpellier, IRD, CNRS, Montpellier, France

iDépartement de Biologie-Ecologie, Faculté des Sciences, Univ Montpellier, Montpellier, France

j Utrecht University, Netherlands

${ }^{k}$ Centre National de la Recherche Scientifique - UMR ASTRE, CIRAD, INRAE - Faculty of Veterinary

Technology, Kasetsart University, Bangkok, Thailand

'Department of Philosophy, YMCA Building, Texas A\&M University, College Station, Texas 77843, USA

${ }^{m}$ Aristotle University of Thessaloniki, 54164, Thessaloniki, Greece

${ }^{n}$ University School for Advanced Study IUSS, Pavia, Italy

*Corresponding authors:

Email address: ddestoum@ifremer.fr (D. Delphine Destoumieux-Garzón)

Email address: franziska.matthies@helmholtz-muenchen.de (F. Matthies-Wiesler) 


\section{ABSTRACT}

The implementation of One Health/EcoHealth/Planetary Health approaches has been identified as key (i) to address the strong interconnections between risk for pandemics, climate change and biodiversity loss, and (ii) to develop and implement solutions to these interlinked crises. As a response to the multiple calls of scientists in that direction, we have put forward seven long term research questions regarding COVID-19 and emerging infectious diseases (EIDs) that are based on an effective integration of environmental, ecological, evolutionary, and social sciences to better anticipate and mitigate EIDs. Research needs cover the social-ecology of infectious disease agents, their evolution, the determinants of susceptibility of humans and animals to infections, and the human and ecological factors accelerating infectious disease emergence. For comprehensive investigation, they include the development of nature-based solutions to interlinked global planetary crises, addressing ethical and philosophical questions regarding the relationship of humans to nature and regarding transformative changes to safeguard the environment and human health. In support of this research, we propose the implementation of innovative multidisciplinary facilities embedded in social-ecosystems locally: the "ecological health observatories" and the "living laboratories". This work has been carried out in the frame of the EC project HERA (www.HERAresearchEU.eu) that aims to set the priorities for an environment, climate and health research agenda in the EU by adopting a systemic approach in the face of global environmental change.

Keywords: One Health, Planetary Health, Pandemics, Ecology, Evolution, Environment, Climate change, Biodiversity loss, Emergence, Pathogen 


\section{Introduction}

The majority of emerging infectious diseases (EIDs), whose incidence has increased over the past decades or threatens to increase in the near future, have a zoonotic origin (Woolhouse and Gowtage-Sequeria, 2005)(Woolhouse et al., 2005)(Jones et al., 2008). Increase is observed both in the frequency of epidemic events and the proportion of zoonotic EIDs, $72 \%$ of which are caused by pathogens originating in wildlife (Jones et al., 2008)(Smith et al., 2014)(Woolhouse et al., 2005). Zoonosis refers to a disease or infection shared between animals - including livestock, wildlife and pets - and humans (World Health Organization. Food and Agriculture Organization of the United Nations. \& World Organisation for animal health, 2019). Such emerging zoonotic events have happened repeatedly in the history of human populations.

Broad scientific consensus suggests that COVID-19 whose etiological agent is SARS-CoV-2 (Severe Acute Respiratory Syndrome Coronavirus 2), has a zoonotic origin. Based on phylogenetic analysis of currently known virus sequences, it is considered that SARS-CoV-2 has probably emerged from a coronavirus present in China and other Asian countries and its closest relative is hosted by horseshoe bats (Latinne et al., 2020; Zhou et al., 2020). However, unlike rabies, Ebola or West Nile virus, the disease is now characterized by strains of SARS-CoV-2 that circulate among human populations, independently of animal reservoirs. It has become a contagious, from person-to-person, disease agent. In that sense, it can be compared to human measles virus (MeV), which emerged from the Rinderpest virus (RDV) harbored by different mammal species 2,400 years ago during the classical Antiquity, and well before the previously estimated 11th century date (Düx et al., 2020), or to Human Immunodeficiency Virus (HIV), which emerged from simian immunodeficiency virus (SIV) infecting non-human primates during the 20th century (Hemelaar, 2012). It is unknown at this stage if SARS-CoV-2 passed through an animal host other than horseshoe bats before infecting humans (Lu et al., 2020). In 2019, a significant number of initial cases $(66 \%)$ was connected to a local market selling seafood and wildlife products in Wuhan, China (Huang et al., 2020). It then spread in China before it crossed borders, and eventually became pandemic within a few months. Its global spread was accelerated by international transportation and travel. In most countries, the pandemic revealed important weaknesses in pandemic preparedness, although we have been warned several times by similar threats over the past decades with the emergence and spread of many viruses such as HIV, Nipah virus, avian influenza type A viruses, and since 2002, SARS-CoV-1 (causing SARS), Middle Eastern Respiratory Syndrome - Coronavirus (MERS), Zika, chikungunya and Ebola viruses.

As human infections are naturally transmitted from animal hosts, zoonotic EIDs are likely to occur repeatedly. Indeed, a majority of the known viruses has been found in both human and at least one other mammal species, indicating they have the potential to become zoonotic (Olival $e t$ al., 2017). Moreover it has been estimated that several hundred thousand mammalian viruses are still to be discovered (Anthony et al., 2013). Others have estimated that there are around 1.7 million viruses from 25 high-risk viral families yet to be discovered in mammals and birds, of which about 700,000 are predicted to have zoonotic potential (Carroll et al., 2018). However, most of these studies tend to focus on viruses as single disease risk parameters without accounting for exposure and vulnerability factors that are paramount to estimate infection risk (Hosseini et al., 2017). Zoonotic disease emergence requires direct or indirect contact between an animal host and humans (Plowright et al., 2017), the microbe needs to be infective for humans, and human habits must favor the transmission and the spread of the pathogen. Known 
drivers of zoonotic disease emergence such as agricultural land conversion and developmental activities, leading to ecosystem degradation, wildlife trade, intensive farming, change in climate patterns, international travel, trade and commerce are constantly accelerating. Not surprisingly EID events have risen significantly over time, with different drivers, land use change and associated practices representing the main lever for them (Guégan et al., 2020) (Jones et al., 2008). This trend is likely to continue and possibly to intensify if drivers are not mitigated (Smith et al., 2014).

Since SARS-CoV-2 emerged, understandably, most research effort has focused on the immediate pandemic response to the virus and on short-term control strategies. Emerging research needs on the interactions between the COVID-19 pandemic and global environmental change have been identified and described (Barouki et al., 2021). Additional questions arise concerning mechanisms that have led to SARS-CoV-2 emergence and that contribute to the emergence of new, potentially more transmissible and/or more virulent variants. Research effort is needed also in the middle and long term to be able to prevent, mitigate and prepare for future pandemics (Guégan et al., 2020). Much of this long-term research relies on often neglected environmental, social-ecological and evolutionary research needs (Roche et al., 2020) and possibly on research-action's frameworks development.

The aim of this document is to identify and put forward important long term research questions that build on and connect to the previously compiled emerging research needs (Barouki et al., 2021). The scope was broadened to include EIDs more generally and also ethical, philosophical, societal and transformational aspects, in order to allow mitigation, the development of predictive scenarios, prevention and better preparedness for and response to potential future pandemic risks. This work has been carried out in the frame of the EC project HERA ${ }^{1}$ (www.HERAresearchEU.eu) that aims to set the priorities for an environment, climate and health research agenda in the EU by adopting a systems approach in the face of global environmental change (GEC). A COVID-19 working group was formed among HERA partners together with scientists specialized in relevant areas to formulate, collect and discuss which long-term research questions arise from the current COVID-19 pandemic, ultimately to reduce the risks of future pandemic emergences and related disastrous effects.

Here, we discuss seven main areas of long term research needs that will have to be addressed by multi-disciplinary and participatory approaches (Figure 1). An international research and implementation network is proposed to bring together these seven main areas, to allow changes of perspectives as well as new research structures, including open access to information and results along the research process to accelerate pre-emptive policy making and implementation of actions aiming at enhanced prevention and preparedness.

\footnotetext{
${ }^{1}$ an EU H2020 programme that includes 24 partner institutes across Europe and hundreds of collaborating researchers and wider community stakeholders
} 


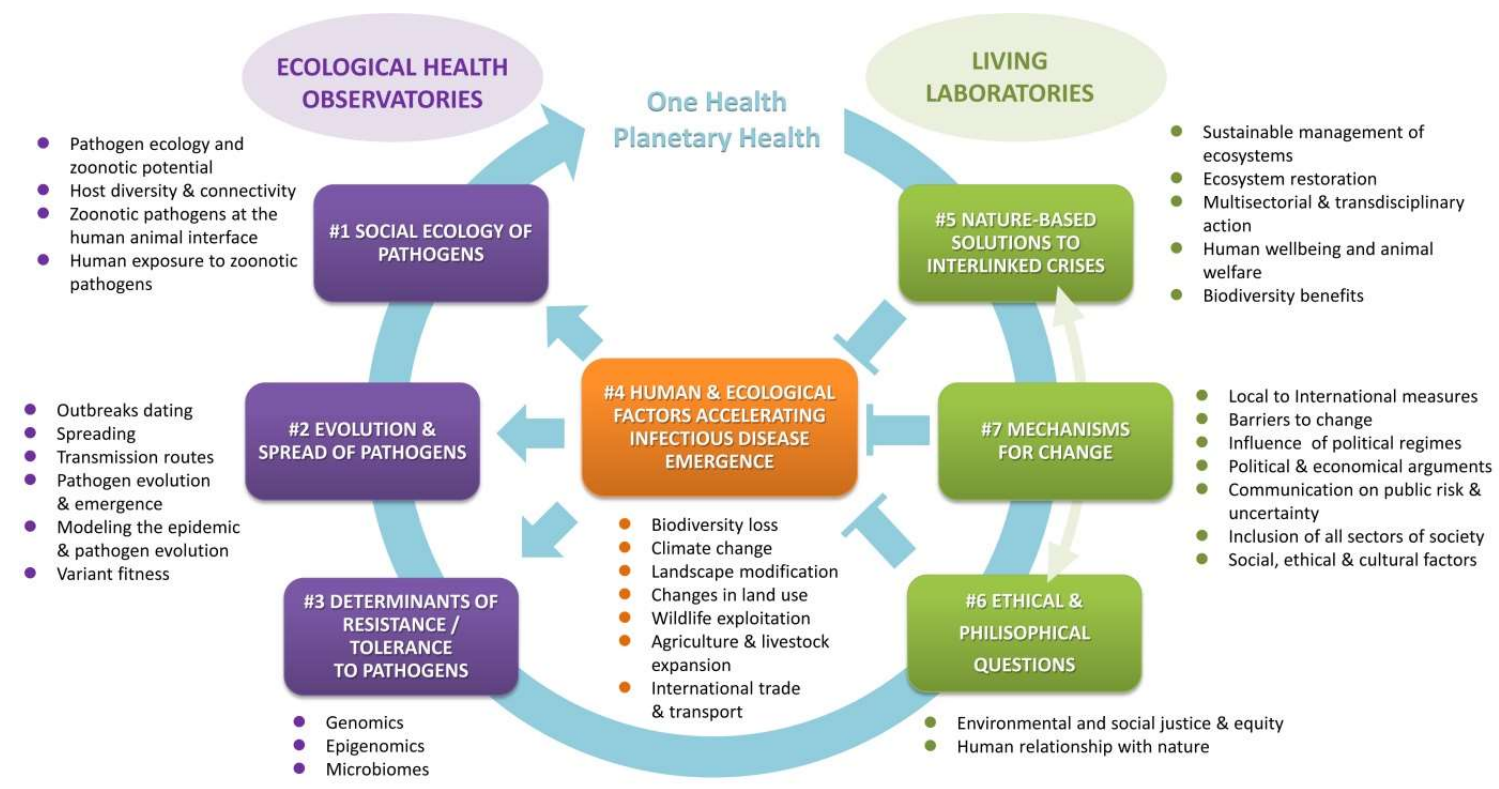

Fig. 1. Mapping of long-term research areas in relation to future risks of pandemics.

Research areas 1-3 investigate key determinants of infectious disease emergence, research area 4 tackles causes and drivers of future pandemics, research areas 5-7 investigates ways towards prevention of future pandemics. Operational facilities are proposed such as ecological health observatories and living laboratories in support of the proposed research. These could be linked through a global network of research centers providing foresight and supporting prevention and preparedness in view of future pandemics facilitating data exchange and validation, interoperability and applicability of computational tools addressing the environmental health risk of pandemics.

\section{Long Term Research Needs \#1: The Social-Ecology of SARS-CoV-2 and Other Pathogens}

Mitigation and prevention strategies require an understanding of what pathogens (e.g., viruses, bacteria, eukaryotes, prions) circulate in ecosystems, and how and why they succeed in crossing species barriers. This calls for a better knowledge of the ecology of pathogens and their transmission routes. A much larger number of wildlife, and livestock samples need to be analyzed for various pathogens, including viruses from high-risk viral families, which could e.g. feed into risk ranking frameworks and web tools such as SpillOver: Viral Risk Ranking (https://spillover.global) (Grange et al., 2021). For that, we need field observations developing research and data acquisition, supported by complex risk and data analysis platforms implementing foresight methodologies to capture and quantify the resulting environmental health risk ahead of time.

\subsection{What do the dynamics of zoonotic pathogens at the human-animal interface look like?}

The Covid-19 pandemic has made clear that it is highly important to characterize the reservoirs of pathogens in areas where human populations and livestock live in close contact with wildlife and/or where human exposure and vulnerability is documented (e.g., where human or environmental perturbations occur; see Guégan et al. (2020) for a recent mapping). This goes beyond a simple survey of SARS-CoV-2 in wild fauna, livestock, pets, water and air, and extends to the diverse pathogens circulating in ecosystems. Monitoring the temporal and spatial patterns of pathogen occurrence in natural land, agricultural, urban and other human-dominated 
ecosystems is absolutely urgent to gain a better knowledge of disease ecology capable of contributing to risk assessment and management. The importance of having ecological literacy on the virus circulation for controlling disease transmission could be demonstrated studying the Nipah virus system in Malaysia and Bangladesh, as well as the risk of emergence in other ecosystems regarding socioecological dynamics (Cappelle et al., 2020) (Plowright et al., 2019).

One important operational priority is the establishment of ecological health observatories, as we highlighted earlier (Barouki et al., 2021). Observatories should implement interdisciplinary and intersectoral approaches, with three main objectives: (i) provide robust data for fundamental research on hot-spots of emergence and/or in environments with high levels of perturbations, leading to high risks of emergence, to inform on the effect of both environmental changes and human practices on the emergence; (ii) assist the development and transfer of accurate pathogen monitoring methods that can be deployed at larger scale to monitor pathogens in urban and wild ecosystems (e.g. wastewater-based epidemiology through sewage surveillance, or surface water monitoring at the mouths of rivers) (Larsen and Wigginton, 2020)(Trottier et al., 2020), and (iii) collaborate to share data and co-built projects between local communities, administrations, other stakeholders and multidisciplinary teams of scientists, veterinarians and medical doctors to anticipate and mitigate epidemics. The latter would warrant the employment of formal foresight methodologies enabling us to address within a single, holistic framework the societal, environmental and ecological dynamics and interactions that underpin the emergence of novel pathogens and the respective public health risk management options.

With the objective of discovering novel viruses with zoonotic potential and anticipate future disease emergence, observatories should collect (with dedicated campaigns), archive, share (through open access databases) and explore metagenomic data for viral sequences, as well as immunogenetic data for hosts, which are needed to explore pathogen evolution (long term research need \#2) and uncover the determinants of tolerance/resistance to the virus in animal populations (long term research need \#3), respectively. Observatories could therefore feed ambitious interdisciplinary research including both laboratory and field programs, by providing knowledge on the ecology of viruses: their natural host range (broad versus narrow), their transmission routes (direct versus vector-borne), and their biology, which are critical parameters for estimating their zoonotic potential (Olival et al., 2017). This basic knowledge is also needed to next clarify what human and ecological factors favor and accelerate pathogen spillovers and transmission to humans (long term research need \#4). It can then bring to setting-up "living laboratories" involving rural actors and aiming at change in agricultural practices and environmental management, transitioning to agroecology (long term research need \#7).

Without excluding the rest of the world, ecological health observatories should be implemented in hot-spots of host reservoirs and disease diversity and emergence. Asian countries are particularly concerned with several viruses of animal origin that have emerged over the past few years, in addition to the still high burden of neglected tropical diseases. Similarly, Central America appears important to survey, as it hosts a melting pot between Neoarctic and Neotropical fauna (and thus of potential pathogens from these two origins) and harbors a high bat species diversity that serve as pathogen reservoirs. In addition, northern countries of South America and several countries of Central and East Africa are predicted to host a high number of undiscovered zoonotic viruses (Olival et al., 2017). Such observatories are not necessarily intended to last over time and can move according to the conditions of emergence and spread. 
Moreover, as far as zoonotic agents are concerned, anticipating future EIDs requires expanding ecological research, which primarily investigates single host-pathogen systems, to a multihostmultipathogen research perspective. The role of the interactions between hosts, vectors, and pathogenic and nonpathogenic agents in the dynamics of zoonotic pathogens at the humananimal interface remains incompletely understood. Gortazar et al. (2014) have developed a framework for analysis of pathways and interactions between pathogens and host or vector species. In addition, it is essential to integrate studies of humans, their different levels of exposures and vulnerabilities, i.e., both for single individuals and whole communities, since human activities and practices can influence the potential for infection from wildlife. As social and ecological factors play a key role in the epidemiology of infectious diseases, a systems-based approach that takes into account these key factors is needed (Box. 1). Monitoring the health effects of exposures in both humans and wildlife can be used as an early-warning/sentinel system (Calvignac-Spencer et al., 2012).

BOX 1. Towards a social-ecology of health

A social-ecological system can be defined as an ecological system (composed of independent biological units in interactions) intricately linked with and affected by one or more social systems (Anderies et al., 2004; Ostrom, 2009; Colding and Barthel, 2019). Clearly, EIDs, public and animal health, social responses and governance could be analyzed using a social-ecological health framework as recently advocated by De Garine-Wichatitsky et al. (2021). Dealing with social-ecological systems needs to better assess (i) "the bio-physical environment" including land productivity, soil erosion, climate factors, or biodiversity; (ii) "the socio-ecosystem" comprising land use management and economic development pathways and (iii) "the governance", referring to rights (access and uses), justice and laws (e.g. environmental law, health law) (Ebbesson and Hey, 2013). A social-ecological approach should first describe how local socialeconomy, governance, environment (land use transformation, climate change) and ecology (community and functional ecology) in relation to global trade and governance may explain the emergence of infectious diseases and epidemic outbreaks. Second, assuming that the adaptive process is linking ecological function and the social system, EID forecasts and scenarios can be developed based on the description of this social-ecological health system through the methodological lens of formal foresight approaches and the respective computational tools. Third, mitigation measures can be implemented through participatory processes involving local communities and administrations, stakeholders and interdisciplinary teams of scientists. Social ecology is not only a technical science dealing with technical problems that can be managed by instrumental decisions (Boer, 1984). Social-ecology and health may help policies and laws to adapt, when facing evolving socio-environments such as EIDs, by acknowledging different values, ethics and responsibilities (Morand and Lajaunie, 2019)

\subsection{What is the actual human exposure to zoonotic pathogens and how to measure it?}

As repeated contacts are required for transmission, it is important to determine whether pathogens and reservoir hosts share the same geographic range with humans, and to estimate the risks of contacts and transmission. So far, most evidence for the role of anthropogenic changes, e.g. encroachment into natural habitats, in the emergence of pathogens originating from wildlife is anecdotal or indirect (Gortazar et al., 2014). However more knowledge is needed 
on causal relationships between changes in host population and species dynamics, the presence of some particular animal species in man-made ecosystems or interspecies contact and pathogen emergence in humans. Existing studies in this area are generally limited in scope and typically lack quantitative assessment of human exposure and vulnerability to zoonotic pathogens at the human-animal interface. The best metrics by which to measure exposure still need to be determined.

To define the actual human exposure to zoonotic pathogens, further assessment e.g., by serology, is suggested, leading to a better understanding of the complete chain of emergence. With the objective to both accurately detect positive cases and implement molecular epidemiology, DNA diagnostic methods should be implemented according to the sequence of the epidemic. DNA detection and amplification methods adapted to several matrices, either environmental (water, soil, sewage..) or biological (feces, urine, serum..) need to be set-up before the emergencies arise. These methods have to be developed both on humans and animals. Targeted approaches (e.g. quantitative PCR, digital PCR, pathochips based on gene expression microarrays and in situ micro-sequencing) but also global approaches (i.e. metagenomics, metatranscriptomics) to assess pathogen communities and infer potential interactions need to be developed.

In addition to molecular techniques, contact estimation between human and animals could also be improved in areas endemic for zoonotic diseases through the development of new technologies and the sharing of data obtained. Technologies such as bio-logging, which is increasingly used by both ecologists and farmers to record behavioral, physiological and ecological variables, in both wild and domestic animals (Destoumieux-Garzón et al., 2020), and the use of smartphones and social media networks to monitor movements of human populations (Finger et al., 2016) are certainly promising methodologies to be further developed for research purposes at the interface of epidemiology and ecology. Beyond estimating exposure, in that case contacts between humans and animals, an effective sharing of pathogen sequences circulating in animals (through ecological health observatories) and human populations (through hospitals) would open avenues to monitor pathogen transmission and evolution in nature, an important piece of information needed for infection risk management. This requires governments to establish publicly available repositories of pathogen sequences.

\section{Long Term Research Needs \#2: The Evolution and Spread of SARS-CoV-2}

The SARS-CoV-2 pandemic can be used as a case study to learn from, in order to prevent that future EIDs develop into pandemics. Therefore, there is a critical need to understand how and why SARS-CoV-2 has emerged and spread. A more effective use of evolutionary sciences is also needed to track the emergence of more infectious and /or more virulent variants and to measure the speed of propagation of the virus (Jones and Manrique, 2020). Paradoxically, while the processes of infectious diseases emergence rely on basic evolutionary principles, population biology and evolutionary sciences remain insufficiently considered in health research. Evolutionary sciences are essential to understand the factors leading to the emergence of new pathogens like SARS-CoV-2, the occurrence of new variants e.g. with enhanced transmissibility, virulence or resistance to drugs or vaccines, the variations of compatibilities between populations of hosts and strains of pathogens etc.; they can also provide tools for disease management (Roche et al., 2020). A series of questions related to evolutionary sciences are not yet present in the international research agenda. 


\subsection{Where did SARS-CoV-2 evolve before it infected humans?}

An important study factor is whether reservoir hosts facilitate viral evolution, as this is likely to favor host switches (Latinne et al., 2020). Important field and sequencing research is needed to obtain this key information, which is currently incomplete due to viruses or host associations that have completely evaded human detection to date (see long term research need \#1). This is well exemplified with SARS-CoV-2. Indeed, while scientific evidence supports the animal origin of SARS-CoV-2, we are still ignorant about which animal species it emerged from. A rather ancient recombination event between bat and pangolin variants is suspected (Wang et al., 2021) but this(se) cannot be traced back as the host(s) from which the virus passed to the human species. Screening wild fauna for viral genomes (metagenomics), and implementing open-access databases is necessary to trace the thread of transmission and viral evolution within the intermediary hosts of SARS-CoV-2. As the number of sequences increases, we should be more successful in identifying the origins of SARS-CoV-2, the new variants and other emerging viruses. Thus, a recent phylogenetic study by Lattine et al. (2020), which included 630 coronavirus sequences isolated from China, suggests that the pangolin is less likely to be the origin of SARSCoV-2 than the horseshoe bats (Rhinolophus spp.). Still the time to the most recent common ancestor of SARS-CoV-2 and bat variants is inferred to be 37 years (Wang et al., 2021) and we have an important missing gap to fill to reconstruct the route of transmission from animals to humans. While sequencing the biodiversity of viruses circulating in the wild fauna cannot be used to predict disease emergence, it can definitely help reconstructing transmission routes; a level of information that will also be needed for future EIDs. In addition, phylodynamics makes it possible to date the outbreaks with confidence, rule out some hypotheses (e.g. rumors about SARS-CoV-2 circulation outside China before November 2019), and identify the host taxa and geographic regions that define hotspots of coronavirus evolutionary diversity as done in China by Latinne et al. (2020). Incorporating phylodynamic inferences into predictive models with the help of machine and deep learning techniques appears highly relevant. This could help target coronavirus discovery in wildlife for proactive zoonotic disease surveillance.

\subsection{Can we predict SARS-CoV-2 evolution (without laboratory experiments) and anticipate future pathogen emergences?}

Evolutionary sciences are needed to identify selective pressures that drive the emergence of new variants. Variants spread poorly over the first year of the pandemic, but since late 2020, some have emerged independently and spread very efficiently in different countries (e.g. UK, South Africa, Brazil, USA). This raises a series of important questions. How can we identify variants with a true fitness advantage (Box 2)? Are SARS-CoV-2 variants emerging in chronic patients during a long battle with imperfect immune responses? Will more variants emerge in already partly immunized populations as a consequence of the co-evolutionary race between the virus and the human immune system (antigenic drift)? This may include imperfect vaccines which may select not only for immune-proof viruses but also potentially for more virulent ones. Identifying the drivers of such emergences is required to adapt COVID-19 mitigation strategies and to anticipate the evolutionary potential of SARS-CoV-2 that threatens current vaccination strategies (Barnes et al., 2020; Wibmer et al., 2021; Xie et al., 2021). Part of the questions can be addressed through modeling pathogen evolution (see below). 
BOX 2. The use of evolutionary sciences to characterize variants with fitness advantage

A major challenge in the context of an epidemic is to distinguish variants with a true fitness advantage over their ancestors and variants with little fitness increase, but which gained a stochastic advantage by surfing on an epidemic wave, thanks to founder events or superspreading. A first characteristic of these new variants is to have a significant boost of amino-acid mutations that makes them substitution rate outliers relative to the molecular clock, often with an excess of non-synonymous over synonymous substitutions. A second characteristic is the presence of key mutations with functional effects such as in the Receptor Binding Domain of the Spike protein. Finally, a third characteristic is parallel/convergent evolution with different variants of concern sharing the same mutations (point mutations or indels) that appeared independently in different genetic backgrounds. These three characteristics are known footprints of adaptive evolution and have allowed anticipated detection of variants in countries with significant sequencing efforts and immediate data sharing, such as the COG-UK Consortium. These variants can then be followed in order to verify whether they consistently increase in frequency each time they are introduced in a new place, or whether this increase depends on some environmental conditions (the proportion of immunized hosts, climate etc...). Functional assays in laboratory experiments can subsequently test the effects of key mutations, including affinity to human receptors, antibody avoidance or resistance to vaccines or treatments.

\subsection{What are the challenges of modeling pathogen evolution and the epidemic?}

There is an important research need to build and validate mathematical models, not only to understand the dynamics of the COVID-19 disease, but also SARS-CoV-2 emergence/evolution, virulence and transmission. Ecological, biological, evolutionary, environmental, climatic, physiological, clinical and socio-economic data are needed to both implement and validate models of emergence, transmission and severity of the corresponding health risk. At the beginning of the epidemic, basic biological and medical data were lacking to inform models (e.g. the fraction of asymptomatic cases, the relative infectiousness of asymptomatic individuals, the time between infection and the onset of symptoms, the infectiousness before symptom onset, the effect of age, etc.) (Day et al., 2020). Still, it was expected that the virus would evolve increased transmission and immune resistance (Day et al., 2020); models are now needed to predict what variant traits will confer fitness advantage as the epidemic evolves. The socioeconomic factors associated to SARS-CoV-2 circulation and spread remain also insufficiently characterized.

Modeling SARS-CoV-2 evolution is critical for the success of public health measures, particularly vaccination. It is both a research need and an important challenge. As discussed above, a series of variants carrying mutations on the spike (recognized by the ACE- 2 receptor of host cells) have spread worldwide, as a consequence of an increased transmissibility about one year after the beginning of the pandemic. However, it is still poorly known to what extent mutations that increase transmissibility affect (either positively or negatively) viral replication or virulence. This level of information is needed for modeling viral evolution. It is also unknown to what extent natural selection for antigenic escape mutations will be increasing as a result of viral circulation in human populations largely immunized either naturally, or through vaccination campaigns (Day et al., 2020), and what viral traits (transmissibility, virulence, immune evasion) will become more favorable for the virus as the percentage of immunized hosts increases 
worldwide. Testing these diverse scenarios deserves an important and coordinated and inclusive research effort based on extensive data sharing.

From an epidemiological point of view, we also need prospective scenarios that take into account global changes and political actions. Beyond demographic and epidemiological data, there is an important challenge in incorporating ecological, evolutionary, climatic, social, economic, behavioural and cultural data, which are key factors influencing epidemics into these scenarios (Barouki et al., 2021). Such scenarios are needed to help decide which mitigation measures to adopt. Integrative approaches incorporating a round-trip from the field to the model and back should be promoted to validate epidemiology-based health risk management models. Such research should include the experimental study of mechanisms on systems where we have access to all the parameters, including knowledge of human activities/practices. Designed/implemented at a local scale (a territory), they would ensure a good knowledge of the parameters and engage local actors who can benefit from practical recommendations around the management of the health risk with which they are confronted at the level of their territory.

From the point of view of social sciences, it will also be important to question to what extent epidemiological modeling has actually contributed to taking political decisions, what were the data that decision-makers lacked/missed to enlighten the situation and provide relevant information for health crisis management (beyond epidemiological data, ecological, social, economic, behavioural, cultural would be needed). A study highlighting the "missing" data, both from the perspective of modelers and decision-makers, is needed. Furthermore, adopting a socioecological health framework adopting foresight methodologies will contribute to filling the knowledge gaps for effective decision making, relying on an in-depth understanding of the biological, ecosystem and social dynamics at play. Participatory modeling approaches in complement to epidemiological modeling can contribute to collective management for EID prevention. Social sciences (including citizen science) are needed in the framework of such transdisciplinary approaches.

\section{Long Term Research Needs \#3: The Determinants of Tolerance/Resistance to and Spreading of SARS-CoV-2 in Human and Animal Populations}

The genetics of virus/host compatibility are expected to be a key determinant of COVID-19 epidemiology, whether hosts are human populations or reservoir animal species.

The potential to host and transmit zoonotic pathogens varies according to animal species (see long term research \#1). Mammals, particularly primates, bats and rodents host a high diversity of zoonotic viruses, and the host traits that predict total viral richness have been studied by Olival et al. (2017). Not surprisingly, the proportion of zoonotic viruses per species was found to increase with host phylogenetic proximity to humans, probably due to evolutionarily related species sharing host cell receptors for virus binding (e.g. the ACE-2 receptor for SARS-CoV-2). Understanding how animal reservoirs (particularly bats) tolerate viruses without developing symptoms could be a source of innovation for the development of antiviral therapies (Irving et al., 2021). This calls upon the support of comparative genomics / comparative immunology programs, which are often neglected. The knowledge of the pathogen host range (long term research \#1) is key for developing comparative immunology programs. In addition, more research is needed on the diversity and mechanisms of reservoir host immune systems and the 
pathways that maintain the co-evolutionary balance between host tolerance and resistance and pathogen virulence.

As SARS-CoV-2 has become transmissible from human to human with major variations in severity of the disease observed worldwide and on an individual scale, there is a lot to learn from population genetics on the diversity of human populations regarding their susceptibility to SARS-CoV-2 and their ability to transmit it. Data are already available on the genetic determinants of resistance/susceptibility to SARS-CoV-2. Very recently, genetic determinants of disease severity in humans (infected individuals that develop pathology) have been identified. Factors determining disease severity appear to depend on human evolutionary history (Zeberg and Pääbo, 2020). Population genetics coupled to genome-wide association studies should be promoted to identify genetic determinants of disease severity in human populations (Casanova et al., 2020). Beyond genetically-encoded information, non-genetic information that can cause phenotypic differences between individuals and account for differences in tolerance/resistance to diseases. Two parameters deserve a research effort. First, we still largely ignore how much the structure and function of the microbiota, i.e. the symbiotic microbial cells (bacteria, Achaea, viruses, and eukaryotes) hosted by animal species, accounts for the susceptibility of individuals to infectious diseases. The social interactions (cooperative and competitive behavior) of microorganisms and the many factors that shape variation in their effects on host functions, including defense against pathogens, remain elusive (Figueiredo and Kramer, 2020). Second, a lot remains to be explored on epigenetic mechanisms that mediate the response of individuals to environmental modifications, including exposure to pathogens. Epigenetic modifications are chemical additions to the DNA and histones that are stably maintained and do not change the primary DNA sequence of a genome; they contribute to the modulation of gene expression (Feil and Fraga, 2012). Previous pandemics have taught us that coronavirus infections (MERS-CoV, SARS-CoV) cause important epigenetic modifications in infected populations (Schäfer and Baric, 2017). These changes affect the innate immune system, whose dysregulated response contributes to severe SARS-CoV-2 infections. Based on these findings it was hypothesized that coronaviruses actively modulate the epigenetic landscape of the human genome. It is expected that epigenetic signatures will provide markers for past viral infections, vaccination response, and for the capacity of the innate immune system to fight off coronavirus infections, opening avenues for future research in immuno-epigenetics (Box 3).

BOX 3. Immuno-epigenetics in coronavirus infections.

There is compelling evidence that a dysregulated innate immune response contributes to the clinical presentation of patients with severe COVID-19 infections. Myeloid cells are central players in innate immunity. Their properties are imprinted during their development by modifications in their epigenome and influenced by environmental cues. These epigenetic mechanisms include histone modifications and changes in DNA methylation (reviewed e.g. Mantovani \& Netea, 2020). It was shown that DNA methylation modifications regulate the expression of key immune system genes, in both innate and adaptative immune responses (Obata et al., 2015). Several studies have also shown that DNA methylation changes upon infection with virus, bacteria and parasites. Epigenetic changes that affect the host immune system were reported in populations infected with parasites such as Plasmodium falciparum (Quin et al., 2017) or Schistosoma mansoni (DiNardo et al., 2019) but also bacteria such as Mycobaterium tuberculosis (DiNardo et al., 2019). Changes upon bacterial infections have been reviewed before (Bierne et al., 2012). Aberrant DNA methylation was also observed upon 
several viral infections (e.g. hepatitis (Song et al., 2016), (Okamoto et al., 2014), Human papilloma virus (Jiménez-Wences and Peralta-Zaragoza..., 2014), choriomeningitis virus (Scharer et al., 2013), herpes simplex (Liang et al., 2009), and others. DNA methylation was even used to predict disease progression and fatality of HIV infection (Zhang et al., 2018) (Chen et al., 2019). Concerning viruses that cause respiratory syndromes, distinct epigenetic signatures were observed upon infection with H1N1-09, SARS-CoV, H5N1-VN1203 and MERS-CoV (see Schäfer and Baric (2017) for a detailed review on coronavirus infections and epigenetic changes). Remarkably, MERS-CoV infection resulted in complete DNA methylation of three antigenpresentation-associated gene promoters (HLA-A, B2M, and PDIA3) in-vitro (Menachery et al., 2018). It was hypothesized that the virus actively modulates the epigenetic landscape of the human genome (Gordon et al., 2020).

\section{Long Term Research Need \#4: Human and Ecological Factors Accelerating Infectious Disease Emergence}

Scientific evidence shows that human activities such as overuse of natural resources and pollution are causing climate change, biodiversity loss, land degradation and landscape modifications. In turn, biodiversity loss, expansion of agriculture and livestock, changes in land use (deforestation, mining and infrastructure development) leading to habitat destruction, wildlife exploitation, climate change and climate variability have been hypothesized as direct or indirect factors causing disease emergence and expansion, i.e., epidemics and pandemics including SARS-CoV-2 (Morand, 2020; Sironi et al., 2020; Beyer et al., 2021; Halonen et al., 2021; Morand and Lajaunie, 2021). Indeed, by promoting more frequent and novel contacts between people, domestic animals, wildlife and the potential pathogens it carries via human practices and habits, global change (including GEC) has the potential to locally trigger host shifts, spillovers and to enhance disease transmission (Patz et al., 2004; IPBES, 2020; OECD, 2020). Globally, there is a considerable lack of data from multidisciplinary studies that take into account the multiple human and ecological factors contributing to disease emergence and transmission. A better knowledge of the factors that influence pathogen ecology, transmission and evolution on the one hand, and the host susceptibility on the other hand, is needed to anticipate, prevent and mitigate the emergence and spread of infectious diseases as far as possible to adapt national and international environmental/agricultural/industrial/societal policies, mitigation, preparedness and response.

5.1. What are the effects of human activities on pathogen evolution and transmission to humans and the host susceptibility?

The risk of zoonotic EID is elevated in forested tropical regions experiencing rapid land-use changes, where mammalian biodiversity is high (Allen et al., 2017; Guégan et al., 2020) and where people are highly vulnerable and constitute the most deprived communities today (Guégan et al., 2020). Investigating the role of habitat destruction on the one hand and human activities/behaviors on the other hand is needed to determine how much they have contributed to recent EIDs originating from animal species, including SARS-CoV-2 (Barouki et al., 2021). This research is needed in the long term as it may help anticipating future crises. At a local scale, it is urgent to characterize the social-ecological conditions prone to favor spill-over and transmission (Box 1). 
In a world where livestock expansion is correlated with the emergence of infectious diseases (Morand, 2020), it is also of prime importance to determine to what extent intensive farming contributes to the rapid evolution and spread of infectious agents in promiscuous and genetically highly similar hosts (Mennerat et al., 2010). As recently seen with SARS-CoV-2, reverse zoonosis (i.e. anthropogenic infection of animals) is possible. This virus has indeed infected mink farms in Western Europe, as well as pets and species of the wild fauna, particularly felines (Hosie et al., 2021). In the same way as animal-to-human transmissions, human-to-animal transmissions are likely favored by repeated contacts and the sharing of similar ACE-2 receptors for SARS-CoV-2 between human and animal species (Palmer et al., 2021). Therefore, what is the risk for human and animal health that the virus spreads and evolves in mammals subjected to breeding in dense populations? To some extent, the humankind is an example of a dense population of hosts for the virus, in which it evolves towards more infectious and more virulent variants. This should motivate research on animal host densities and the risk of epidemics.

With the increase in chemical pollution associated with the industrial and post-industrial era, it is also necessary to assess the potential role of water and soil degradation in infectious disease emergence, in decreasing biodiversity (see below), modifying trophic chains, accelerating pathogen evolution (through mutation or recombination) or selecting drug resistance in pathogens and tolerant species to pathogens likely to help to the dispersion of zoonotic diseases. Chemical pollution is likely to increase vulnerability through immune toxicity (including changes in immunoepigenetic landscapes), thereby participating in the emergence of still poorly understood multifactorial diseases. We have earlier discussed the increased risk of infectious diseases as a result of exposure to toxicants in human and animal species when the immune system is directly or indirectly affected (Destoumieux-Garzón et al., 2018). Understanding the effects of toxicants on the viability of human and animal populations and how they can promote the transmission of zoonotic agents by increasing the prevalence of pathogens in animal reservoirs or intermediary hosts is of primary importance to better mitigate EIDs .

\subsection{What are the relationships between climate change, biodiversity loss and infectious} diseases?

As we are confronted with rapid GEC, we also need to better characterize the role of climate change in driving biodiversity loss and disease emergence (Keesing et al., 2010; WHO, 2015; Morand and Lajaunie, 2018; IPBES, 2020). Further characterization of the relationships between environmental conditions, especially climate and weather, and host and/or vector population dynamics, as well as exploration of pathogen survival and propagation in the environment is required (Gortazar et al., 2014). A recent literature review, on links between ecological integrity and EIDs originating from wildlife, points at research gaps, such as the detection and prediction of associations between drivers such as climate change or weather variability and pathogen emergence (Evans et al., 2020). Specifically, investigation is needed into the effects of species diversity and function, disturbance and human-wildlife contacts on emergence and spread of infectious diseases. Biodiversity loss (e.g. extinctions or local extirpations of some species) is considered as a driver of zoonotic agent emergence as it can increase the risk of diseases being transmitted among the remaining species. Indeed, multi-host pathogens are expected to benefit from biodiversity loss. One underlying principle is the dilution effect: as host species can differ 
dramatically in their quality as a reservoir, a larger number of poor-quality reservoir species is expected to dilute events of pathogen transmission, as opposed to a small number of high quality reservoir species, which amplify transmission. This has been documented in vector-borne diseases (Keesing et al., 2009). Moreover, when the number of species decreases, intra-specific density is expected to increase, thereby facilitating transmission among reservoir species. The increase in density of reservoir species can also favor transmission to humans as a consequence of augmented contacts. However, to date there is insufficient evidence to confirm how common 'dilution' and 'amplification' effects are a consequence of biodiversity loss (Keesing et al. 2009, Randolph \& Dobson 2012). Recent evidence tends to suggest that for several disease systems, e.g., cutaneous Leishmaniosis, Hantavirus syndrome, Buruli ulcer/Mycobacterium ulcerans, Lyme disease, both phenomena, i.e., a dilution and an amplification transmission, can happen in space and in time, emphasizing the need for a critical and balanced approach to that question (Luis et al., 2018; Guégan et al., 2021).

\section{Long Term Research Needs \#5: Nature-Based Solutions and Approaches to Interlinked Crises}

Due to the intricate links that exist between climate change, biodiversity loss and disease emergence (both in human and animal populations), nature-based solutions will be required. Nature-based solutions are defined as actions to protect, sustainably manage, and restore natural or modified ecosystems, which address societal challenges effectively and adaptively, simultaneously providing human well-being and biodiversity benefit (Cohen-Shacham, E., Walters, G., Janzen, C. and Maginnis, 2016). Research into effective nature-based solutions is important as spearhead in addressing the ecological and the health crisis jointly. Indeed, beyond reducing the risk of pandemics, nature-based solutions have the potential to reduce the impact of interlinked crises (biodiversity loss, climate change etc.) that affect unequally and inequitably human populations. For instance, protecting tropical forests would have much-needed ancillary benefits for climate protection in turn, natural resources conservation and autochthonous tribes and traditional beliefs as well (Guégan et al., 2020). Strategies and methods to proactively conserving and restoring our remaining intact ecosystems (e.g. forests) to halt climate change, biodiversity loss, and - we must hope - the next pandemic need to be further explored (Oakes, L.E. et al., 2020), as well as ways to implement them. Research to develop, foster and implement multisectorial and trans-disciplinary action in line with the Planetary Health and the One Health approaches needs to be strengthened. Actions and measures need to be implemented, monitored and evaluated at national and often territorial levels. This includes identifying and assessing opportunities and benefits for the lives, activities, well-being and health together with local communities, requiring a strong integration of social sciences in the framework of living laboratories implementation for example. An example for an initiative that can trigger international and multi-sectoral actions is the FAO-OIE-WHO Tripartite guide "Taking a Multisectoral, One Health Approach: A Tripartite Guide to Addressing Zoonotic Diseases in Countries" in 2019 (WHO, FAO \& OIE, 2019), which now involves the UN environment programme (UNEP) in a quadripartite task force (de Garine-Wichatitsky et al., 2020). 


\section{Long Term Research Needs \#6: Ethical and Philosophical Questions Related to The Relationship of Humans and Nature and the Implications For Change}

The current pandemic provides a window through which to examine broader philosophical and ethical questions about human relationships to the non-human world. The emergence of new infectious diseases raises profound questions about the industrial development of wild environments including tropical forests, the use of wildlife, the intensification of animal husbandry, the impacts of climate change, and the promotion of economies and lifestyles that contribute to these effects. If we want to reduce the risk of future pandemics, reconsidering the frameworks of the values that underpin such risk will be necessary.

This kind of reconsideration of values will need to be very broad. It will need to encompass issues of environmental and social justice and equity, grounded in recognition of our mutual interdependence and shared vulnerability (Ruger, 2020) and taking into account, for instance, how far the environmental destruction and wildlife use that increase the risk of EIDs are related to poverty and distributive and participative injustice. Such a reconsideration of value frameworks should also take into account justice between human generations, in particular what is owed by present people to future people with respect to reducing the risk of pandemic disease that they face, as well as what kind of environment and associated society and economy we will be leaving to them.

This reconsideration should move beyond the human to consider human relationships with both wild and domesticated animals (Rock and Degeling, 2015). The questions here are certainly complicated, and are likely to reveal tensions between the values of animal welfare, human food systems, cultural values, and the values of threatened and endangered ecosystems and species. But the complex nature of these ethical questions is an indication both of how important and urgent it is to begin exploring them now, and of the need to make such exploration a key target for long-term research.

Research into ideas about the relationships between humans and nature more generally will be needed. It should address questions such as: Do anthropocentric attitudes of human superiority and separation help to bring about the destruction of nature that increases the risk of pandemics (Crist, 2018)? Does the natural world have "intrinsic value" and what do we mean by this term (Palmer et al., 2014; Dasgupta, 2021)? To what degree should humans intervene in the environment in order to decrease disease risks to humans - for instance, by gene editing organisms so that they pose less risk of transmitting disease? How far do human beliefs, attitudes and values underpin the destructive environmental practices that increase the risk of EIDs - or are other social and political factors more directly important? Humility is needed instead of the assumption that humans can investigate, predict and control all risks emerging from viruses, bacteria and other microorganisms on Earth, as discussed in research need \# 2 . Here again, interactions with the framework mobilized for transitioning toward agroecology could be addressed at Science/Society interface with perspectives in terms of theory of change.

This kind of research could be pursued within One Health and Planetary Health ethical frameworks that recognize the ways in which concerns about public health, climate change, biodiversity loss, environmental justice and animal welfare are inextricably entwined (Destoumieux-Garzón et al., 2018; Gruetzmacher et al., 2021) and that attempt to tackle these issues collaboratively and across disciplines, drawing on expertise not only from medicine and 
the natural sciences, but also from ethics, law, religion, indigenous knowledge, behavioural economics, psychology and sociology.

\section{Long Term Research Needs \# 7: Mechanisms for the Implementation of Profound Changes to Safeguard the Environment and Human Health and to Reduce Future Risks of EIDs}

The linkages between the integrity of ecosystems and emerging zoonotic diseases still requires further research (Long term Research Need \#1) and in the shorter term, the precautionary principle necessitates that strong action is taken while this additional research is undertaken (Evans et al., 2020).

Research reviewed in the global report of the Intergovernmental Science-Policy Platform on Biodiversity and Ecosystem Services (IPBES) in 2019 suggests that there is growing knowledge available that provides a pathway to predicting and preventing pandemics (IPBES, 2019). The connections between land use and climate change as key drivers of biodiversity loss and the links to disease emergence and pandemic risks provide opportunities for prevention, control and response measures (IPBES, 2020). Therefore, change towards sustainability requires addressing these root causes, implying fundamental changes in society (IPBES, 2019; HERA WP3 results). IPBES has put forward potential policy options that support transformational changes to reduce pandemic risks and potentially prevent future pandemics (IPBES, 2019, 2020; Box 4).

To achieve transformational change and the implementation of recommended policy options, trans-disciplinary implementation research involving social sciences is particularly needed on: i) the social, ethical, psychological and cultural factors influencing behavioral change in times of a national/international health crisis; ii) improving the link from science to policy to public risk communication and dialogue on the need for transformational change towards sustainable development, considering the lessons learnt from the COVID-19 pandemic (Ref. to WP3/ Workshop results; forthcoming); iii) the most effective way of communicating uncertainty between and beyond disciplines to donors and policy makers; iv) the links between gender, environment, exposure and disease as well as social factors and exposure; v) the multiple barriers to change and the identification of leverage points for transformational change and vi) the influence of types of governance to influence transformation. "Living laboratories" bring together public and private actors, companies, associations and individuals, with the aim of testing new services, tools or uses in real life. They may be defined as a user-centered, iterative, open-innovation ecosystem, often operating in a territorial context (e.g. city, agglomeration, region or campus), integrating concurrent research and innovation processes within a publicprivate-people partnership. They are a potential format for including all sectors of society and for allowing innovative practices to emerge. Results can potentially provide good practice examples and identify and characterize technological and social feasibility and limitations as well as spatial and ecological conditions of transformational changes involving nature and people need to be further investigated (IPBES, 2019).

The results are required, for example, to inform concerted international and national action, integrating low- and middle-income countries (LMICs) to improve pandemic prevention, preparedness and response. A planetary health approach needs to focus on reducing inequalities, including the potential of including women in decision making, especially in LMICs 
(de Paula et al., 2021). A foresight paradigm encompassing both participatory methodologies and foresight computational tools could further enhance our capacity to capture the social, economic, environmental, ecological dynamics that determine the emergence of infectious health risks in different socio-economic and cultural contexts.

BOX 4. Policy options to reduce pandemic risks (IPBES, 2019)

Topics include: setting up enabling mechanisms; increasing sustainability and reducing pandemic risk due to land use change and agricultural expansion; monitoring and protecting "protected areas" including through legal frameworks; and reducing pandemic risk due to the wildlife trade, and in this context, emphasizing high quality alternatives to wild meat, particularly banning or replacing luxury bushmeat in urban areas, while fully respecting the legitimate rights of Indigenous Peoples and Local Communities who still depend on wildlife as a vital source of food, income and cultural identity.

Indeed, it is usually the hunting and dismembering that poses the higher risk for pathogen transmission. However, the 'luxury bushmeat' sold in urban areas, will have been hunted and dismembered, and for every bushmeat item sold there was a hunter in contact with an animal (their secretions and possibly getting scratched/wounded); which adds another layer, that of injustice, because it puts the hunters and people from poorer rural areas at higher risk, to satisfy non-essential demand in urban areas. Also, despite few studies of the mechanisms that drive risk, recent data demonstrate that the percentage of bamboo rats infected by coronaviruses increases through the wildlife trade value chain in Vietnam, from $6 \%$ in rat farms, to $21 \%$ in large live animal markets, to $56 \%$ at the point of slaughter in restaurants. Coronavirus testing indicates transmission risk increases along wildlife supply chains for human consumption in Viet Nam, 2013-2014 (Huong et al., 2020).

Economic arguments are important when investigating and discussing options for the implementation of ambitious policies that safeguard nature (tackling climate change and biodiversity loss), strengthen the resilience of health systems, improve the protection of health and support the reduction of inequalities (WHO, 2020). With respect to reducing the risks of pandemics, comparing economic assessment of the disease and of the prevention plans is an important aspect to be considered in health and environmental impact assessments and the development and improvement of respective impact assessment tools. It was estimated in 2020 that the COVID-19 pandemic would likely cost between US\$8.1 and US\$15.8 trillion globally. Dobson et al. (2020) estimated that in comparison, the present value of prevention costs for 10 years amount to only about $2 \%$ of the current pandemic cost: a significant reduction in transmission of new diseases from tropical forests would cost about US\$25 billion/year. It needs to be investigated how to successfully make the case for a green, healthy and just recovery for people and the planet and to focus on what is driving human health and well-being (Leggewie, 2020). This includes exploring the transformational potentials of a "Great Mindshift" towards mainstreaming economics for the agenda of sustainable development (Göpel, 2016).

\section{Discussion And Conclusions}

"The problems of pandemics, climate and biodiversity loss are strongly interconnected - but so are the solutions" (Oakes et al., 2020). These massive crises share the destructive relationship of 
most humans to nature as a core cause. It is important to use a new framework to identify and quantify associations, causal relationships, and risks between ecological change and pathogen emergence (Gortazar et al., 2014). In line with this statement and the multiple calls for a more integrated action on Health, we have identified seven long research needs that promote an effective integration of environmental, ecological, evolutionary, and social sciences to better anticipate and mitigate EIDs. Together with innovative multidisciplinary facilities embedded in social-ecosystems, the "ecological health observatories" and the "living laboratories", they should respond to an increasing demand of society for a sustainable management of ecosystems of which the human species is an integral part and from which it strongly depends.

Various recommendations for healthy and green recovery from the current COVID-19 pandemic have been put forward, on the basis of what is known today about interconnections, drivers and consequences. The World Health Organization, for example, has compiled a set of prescriptions and actions for a healthy and green recovery from the COVID-19 pandemic (World Health Organization, 2020): protecting and preserving nature as the source of human health; investing in essential services such as water and sanitation and clean energy; ensuring a quick and healthy energy transition; promoting healthy and sustainable food systems and building healthy, livable cities and divestment from harmful activities. They are in line with what has been suggested by the OECD in its report entitled "Biodiversity and the economic response to COVID-19: Ensuring a green and resilient recovery" (OECD, 2020), namely "to i) ensure that COVID-19 economic recovery measures do not compromise biodiversity; (ii) scale up investment in biodiversity conservation, sustainable use and restoration; (iii) put a price on biodiversity loss; and (iv) foster cross-sectoral and to integrate international collaboration by adopting the One Health approach, supporting developing countries to safeguard their biodiversity, and developing an ambitious post-2020 global biodiversity framework". , overall, it has been estimated that the cost of the response to COVID-19 will be significantly higher than that of prevention (Dobson et al., 2020). In addition, the pandemic has increased poverty and hunger, and it threatens global stability (Omtzigt and Pople, 2020). The stimulus measures to support the economies around the globe announced were in the order of USD 10 trillion (OECD, 2020). These amounts are potentially powerful levers towards a healthy recovery when invested in sectors and measures that, respectively, safeguard nature, strengthen the resilience of health systems, improve the protection of health and support the reduction of inequalities (WHO, 2020), towards achieving the SDGs.

All of these activities are linked to further long term research as well as the need for urgent and immediate action. Lessons from the COVID-19 pandemic can help to tackle future pandemics as well as other global emergencies (Vinke et al., 2020). Recovery measures will need to be assessed for their short and long term effects on the drivers of potential pandemics and it is important to keep track of constantly emerging results of research on COVID-19 and global environmental change to be able to link them to new research questions aligned with societal needs. To ensure multi-disciplinary, intrediscplinary, cross-sectoral and participatory research to answer emerging questions in relation to pandemic risk and environmental change, we propose setting up an international scientific and strategic network of scientists and stakeholders to jointly work towards the prevention of, preparedness and response to potential EIDs. Open access to and exchange of emerging knowledge is key for an accelerated translation of knowledge into policy and practice. 
In agreement with the conclusion of (Barouki et al., 2021) for emerging research needs in the context of COVID-19 and the environment, mid- and long term research into EIDs and environmental change will also need to adopt an integrative approach with the aim of reducing the risks of future pandemics. Research efforts need to be translated into solutions to jointly protect and restore ecosystems and biodiversity in order to protect human health, including from potential EIDs (WHO, 2015). The key justifications for comprehensive transformative action to safeguard the planet and human health lie in the planetary boundaries and the interactions between the Earth system's processes (Steffen et al., 2015; Lade et al., 2020). Supporting a One Health and Planetary Health scientific research approach, linking human, animal and ecosystem health to design and test better strategies to prevent pandemics, and an agreement to strive for aims and goals of these approaches would be required (IPBES, 2020). We must not lose sight of the bigger challenges ahead in view of the COVID-19 pandemic: In a world where the human population is expanding at unprecedented rates, the excess consumption of resources goes far beyond what planet Earth can cope with; human activities are also driving global warming and the loss of biodiversity, two major threats for life on our planet. Tackling the crises jointly holds unique opportunities to rethink current economic models as well (Halonen et al., 2020, 2021; WHO, 2020).

In the context of EIDs and environmental change - as for other challenges we face to safeguard livable conditions - research needs to be consequential and solution oriented. It has to embrace the complexity of the issues addressed and focus on the most vulnerable (Frumkin, 2015). Acting and advocating with a level of urgency proportionate to the scale of the threat, adhering to the best available science, and practicing clear and consistent communications, are principles that apply to critical health crises like the COVID-19 pandemic and global climate change alike (Watts et al., 2021). To reach these ambitious long term goals, the set-up of an international scientific and strategic network for the prevention of, preparedness for and response to EIDs, including researchers, stakeholders and decision makers could lead the way, to foster the transdisciplinary and cross-sectoral collaboration that is required. Offering a discussion platform and open access and exchange of emerging knowledge and solutions can accelerate the translation of research into policy and practice in this regard.

\section{Acknowledgements}

The authors acknowledge the HERA project that has received funding from the European Union's Horizon 2020 research and innovation programme under grant agreement $\mathrm{N} \circ 825417$. DDG and JB received support from the "Laboratoires d'Excellence (LABEX)" Tulip (ANR-10LABX-41). JFG has benefited from one "Investissement d'Avenir" grant managed by Agence Nationale de la Recherche (CEBA: ANR-10-LABX-25-01). The National Institutes of Health National Science Foundation Ecology of Infectious Disease Program Grant NSF-1911457 provided support to J-FG. J-FG is also supported by IRD, INRAE, the French School of Public Health (EHESP) and University of Montpellier. S.M. is supported by French ANR FutureHealthSEA (ANR-17-CE35-0003-01) and the Thailand International Cooperation Agency (TICA) project "Animal Innovative Health". We are grateful to Dr. Ana Rivero (CNRS, France) for critical reading of the manuscript. 


\section{References}

Allen, T., Murray, K.A., Zambrana-Torrelio, C., Morse, S.S., Rondinini, C., Di Marco, M., et al. (2017) Global hotspots and correlates of emerging zoonotic diseases. Nat. Commun. 8: 1-10.

Anderies, J.M., Janssen, M.A., and Ostrom, E. (2004) A Framework to Analyze the Robustness of Social-ecological Systems from an Institutional Perspective. Ecol. Soc. 9:.

Anthony, S.J., Epstein, J.H., Murray, K.A., Navarrete-Macias, I., Zambrana-Torrelio, C.M., Solovyov, A., et al. (2013) A strategy to estimate unknown viral diversity in mammals. MBio 4: 1-15.

Barnes, C.O., Jette, C.A., Abernathy, M.E., Dam, K.M.A., Esswein, S.R., Gristick, H.B., et al. (2020) SARS-CoV-2 neutralizing antibody structures inform therapeutic strategies. Nature 588: 682-687.

Barouki, R., Kogevinas, M., Audouze, K., Belesova, K., Bergman, A., Birnbaum, L., et al. (2021) The COVID-19 pandemic and global environmental change: Emerging research needs. Environ. Int. 146:.

Beyer, R.M., Manica, A., and Mora, C. (2021) Shifts in global bat diversity suggest a possible role of climate change in the emergence of SARS-CoV-1 and SARS-CoV-2. Sci. Total Environ. 767: 145413.

Boer, B. (1984) Social ecology and environmental law. Environ. Plan. Law J. 1: 233-257.

Calvignac-Spencer, S., Leendertz, S.A.J., Gillespie, T.R., and Leendertz, F.H. (2012) Wild great apes as sentinels and sources of infectious disease. Clin. Microbiol. Infect. 18: 521-527.

Cappelle, J., Hoem, T., Hul, V., Furey, N., Nguon, K., Prigent, S., et al. (2020) Nipah virus circulation at human-bat interfaces, cambodia. Bull. World Health Organ. 98: 539-547.

Carroll, D., Daszak, P., Wolfe, N.D., Gao, G.F., Morel, C.M., Morzaria, S., et al. (2018) The Global Virome Project. Science (80-. ). 359: 872-874.

Casanova, J.L., Su, H.C., Abel, L., Aiuti, A., Almuhsen, S., Arias, A.A., et al. (2020) A Global Effort to Define the Human Genetics of Protective Immunity to SARS-CoV-2 Infection. Cell 181: 1194-1199.

Cohen-Shacham, E., Walters, G., Janzen, C. and Maginnis, S. ed. (2016) Nature-based solutions to address global societal challenges IUCN, Gland, Swizerland.

Colding, J. and Barthel, S. (2019) Exploring the social-ecological systems discourse 20 years later. Ecol. Soc. 24:

Crist, E. (2018) Re-Imagining the Human.

Dasgupta, P. (2021) The Economics of Biodiversity: The Dasgupta review London: HM Treasury.

Day, T., Gandon, S., Lion, S., and Otto, S.P. (2020) On the evolutionary epidemiology of SARS-CoV2. Curr. Biol. 30: R849-R857.

Destoumieux-Garzón, D., Bonnet, P., Criscuolo, F., Henry, P., Prunet, P., Salvat, G., et al. (2020) OneARK : Strengthening the links between animal production science and animal ecology. Animal.

Destoumieux-Garzón, D., Mavingui, P., Boetsch, G., Boissier, J., Darriet, F., Duboz, P., et al. (2018) The one health concept: 10 years old and a long road ahead. Front. Vet. Sci. 5:. 
Dobson, A.P., Pimm, S.L., Hannah, L., Kaufman, L., Ahumada, J.A., Ando, A.W., et al. (2020) Ecology and economics for pandemic prevention. Science (80-. ). 369: 379-381.

Düx, A., Lequime, S., Patrono, L.V., Vrancken, B., Boral, S., Gogarten, J.F., et al. (2020) Measles virus and rinderpest virus divergence dated to the rise of large cities One Sentence Summary: HHS Public Access. Science (80-. ). 368: 1367-1370.

Ebbesson, J. and Hey, E. (2013) Introduction: Where in law is social-ecological resilience? Ecol. Soc. 18: 1-4.

Evans, T., Olson, S., Watson, J., Greutzmacher, K., Pruvot, M., Jupiter, S., et al. (2020) Links between ecological integrity, emerging infectious diseases and other aspects of human health - an overview of the literature.

Feil, R. and Fraga, M.F. (2012) Epigenetics and the environment: Emerging patterns and implications. Nat. Rev. Genet. 13: 97-109.

Figueiredo, A.R.T. and Kramer, J. (2020) Cooperation and Conflict Within the Microbiota and Their Effects On Animal Hosts. Front. Ecol. Evol. 8:.

Finger, F., Genolet, T., Mari, L., De Magny, G.C., Manga, N.M., Rinaldo, A., and Bertuzzo, E. (2016) Mobile phone data highlights the role of mass gatherings in the spreading of cholera outbreaks. Proc. Natl. Acad. Sci. U. S. A. 113: 6421-6426.

Frumkin, H. (2015) Towards consequential environmental epidemiology, Commentary,. Epidemiology 26:.

de Garine-Wichatitsky, M., Binot, A., Morand, S., Kock, R., Roger, F., Wilcox, B.A., and Caron, A. (2020) Will the COVID-19 crisis trigger a One Health coming-of-age? Lancet Planet. Heal. 4: e377-e378.

De Garine-Wichatitsky, M., Binot, A., Ward, J., Caron, A., Perrotton, A., Ross, H., et al. (2021) "Health in" and "Health of" Social-Ecological Systems: A Practical Framework for the Management of Healthy and Resilient Agricultural and Natural Ecosystems. Front. Public Heal. 8: 1-15.

Göpel, M. (2016) How to Work a Great Mindshift for Sustainability Transformations.

Gortazar, C., Reperant, L.A., Kuiken, T., de la Fuente, J., Boadella, M., Martínez-Lopez, B., et al. (2014) Crossing the Interspecies Barrier: Opening the Door to Zoonotic Pathogens. PLoS Pathog. 10:.

Grange, Z.L., Goldstein, T., Johnson, C.K., Anthony, S., Gilardi, K., Daszak, P., et al. (2021) Ranking the risk of animal-to-human spillover for newly discovered viruses. Proc. Natl. Acad. Sci. 118: e2002324118.

Gruetzmacher, K., Karesh, W.B., Amuasi, J.H., Arshad, A., Farlow, A., Gabrysch, S., et al. (2021) The Berlin principles on one health - Bridging global health and conservation. Sci. Total Environ. 764:.

Guegan, J.-F., Roche, B., and Morand, S. (2021) Biodiversity and human health: Go back to the basics of medicine and public health! In, Michel Loreau,A.H. and F.I. (ed), The ecological and societal consequences of biodiversity loss. ISTE Editions, London, UK.

Guégan, J.F., Ayouba, A., Cappelle, J., and De Thoisy, B. (2020) Forests and emerging infectious diseases: Unleashing the beast within. Environ. Res. Lett. 15:. 
Halonen, J.I., Erhola, M., Furman, E., Haahtela, T., Jousilahti, P., Barouki, R., et al. (2021) A call for urgent action to safeguard our planet and our health in line with the helsinki declaration. Environ. Res. 193:.

Halonen, J.I., Erhola, M., Furman, E., Haahtela, T., Jousilahti, P., Barouki, R., et al. (2020) The Helsinki Declaration 2020: Europe that protects. Lancet Planet. Heal. 4: e503-e505.

Hemelaar, J. (2012) The origin and diversity of the HIV-1 pandemic. Trends Mol. Med. 18: 182192.

Hosie, M.J., Hofmann-Lehmann, R., Hartmann, K., Egberink, H., Truyen, U., Addie, D.D., et al. (2021) Anthropogenic Infection of Cats during the 2020 COVID-19 Pandemic. Viruses 13: $1-13$.

Hosseini, P.R., Mills, J.N., Prieur-Richard, A.H., Ezenwa, V.O., Bailly, X., Rizzoli, A., et al. (2017) Does the impact of biodiversity differ between emerging and endemic pathogens? The need to separate the concepts of hazard and risk. Philos. Trans. R. Soc. B Biol. Sci. 372:.

Huang, C., Wang, Y., Li, X., Ren, L., Zhao, J., Hu, Y., et al. (2020) Clinical features of patients infected with 2019 novel coronavirus in Wuhan, China. Lancet 395: 497-506.

Huong, N.Q., Nga, N.T.T., van Long, N., Luu, B.D., Latinne, A., Pruvot, M., et al. (2020) Coronavirus testing indicates transmission risk increases along wildlife supply chains for human consumption in Viet Nam, 2013-2014. PLoS One 15: 2013-2014.

IPBES (2019) Summary for policymakers of the global assessment report on biodiversity and ecosystem services of the Intergovernmental Science-Policy Platform on Biodiversity and Ecosystem Services E. S. Brondizio, J. Settele, S. Díaz, and H.T.N. (ed) Bonn, Germany.

IPBES (2020) Workshop Report on Biodiversity and Pandemics of the Intergovernmental Platform on Biodiversity and Ecosystem Services. Daszak, P., Amuasi, J., das Neves, C. G., Hayman, D., Kuiken, T., Roche, B., Zambrana-Torrelio, C., Buss, P., Dundarova,H. (ed) Bonn, Germany.

Irving, A.T., Ahn, M., Goh, G., Anderson, D.E., and Wang, L.F. (2021) Lessons from the host defences of bats, a unique viral reservoir. Nature 589: 363-370.

Jones, K.E., Patel, N.G., Levy, M.A., Storeygard, A., Balk, D., Gittleman, J.L., and Daszak, P. (2008) Global trends in emerging infectious diseases. Nature 451: 990-993.

Jones, L.R. and Manrique, J.M. (2020) Quantitative phylogenomic evidence reveals a spatially structured SARS-CoV-2 diversity. Virology 550: 70-77.

Keesing, F., Belden, L.K., Daszak, P., Dobson, A., Harvell, C.D., Holt, R.D., et al. (2010) Impacts of biodiversity on the emergence and transmission of infectious diseases. Nature 468: 647652.

Keesing, F., Brunner, J., Duerr, S., Killilea, M., LoGiudice, K., Schmidt, K., et al. (2009) Hosts as ecological traps for the vector of Lyme disease. Proc. R. Soc. B Biol. Sci. 276: 3911-3919.

Lade, S.J., Steffen, W., de Vries, W., Carpenter, S.R., Donges, J.F., Gerten, D., et al. (2020) Human impacts on planetary boundaries amplified by Earth system interactions. Nat. Sustain. 3: 119-128.

Larsen, D.A. and Wigginton, K.R. (2020) Tracking COVID-19 with wastewater. Nat. Biotechnol. 38: 1151-1153. 
Latinne, A., Hu, B., Olival, K.J., Zhu, G., Zhang, L., Li, H., et al. (2020) Origin and cross-species transmission of bat coronaviruses in China. Nat. Commun. 11:

Lu, R., Zhao, X., Li, J., Niu, P., Yang, B., Wu, H., et al. (2020) Genomic characterisation and epidemiology of 2019 novel coronavirus: implications for virus origins and receptor binding. Lancet 395: 565-574.

Luis, A.D., Kuenzi, A.J., and Mills, J.N. (2018) Species diversity concurrently dilutes and amplifies transmission in a zoonotic host-pathogen system through competing mechanisms. Proc. Natl. Acad. Sci. U. S. A. 115: 7979-7984.

Mennerat, A., Nilsen, F., Ebert, D., and Skorping, A. (2010) Intensive Farming: Evolutionary Implications for Parasites and Pathogens. Evol. Biol. 37: 59-67.

Morand, S. (2020) Emerging diseases, livestock expansion and biodiversity loss are positively related at global scale. Biol. Conserv. 248: 108707.

Morand, S. and Lajaunie, C. (2019) Linking biodiversity with health and wellbeing: consequences of scientific pluralism for ethics, values and responsibilities. Asian Bioeth. Rev. 11: 153-168.

Morand, S. and Lajaunie, C. (2018) Loss of Biological Diversity and Emergence of Infectious Diseases. In, Biodiversity and Health., pp. 29-47.

Morand, S. and Lajaunie, C. (2021) Outbreaks of Vector-Borne and Zoonotic Diseases Are Associated With Changes in Forest Cover and Oil Palm Expansion at Global Scale. Front. Vet. Sci. 8: 661063.

Oakes, L.E., Olson, S.H., and Watson, J. (2020) Preventing pandemics, global warming and environmental degradation all at once. CNN.

OECD (2020) Biodiversity and the economic response to COVID-19 : Ensuring a green and resilient recovery.

Olival, K.J., Hosseini, P.R., Zambrana-Torrelio, C., Ross, N., Bogich, T.L., and Daszak, P. (2017) Host and viral traits predict zoonotic spillover from mammals. Nature 546: 646-650.

Ostrom, E. (2009) A general framework for analyzing sustainability of social ecological systems. Science (80-. ). 325: 419-422.

Palmer, C., McShane, K., and Sandler, R. (2014) Environmental ethics. Annu. Rev. Environ. Resour. 39: 419-442.

Palmer, M. V., Martins, M., Falkenberg, S., Buckley, A., Caserta, L.C., Mitchell, P.K., et al. (2021) Susceptibility of white-tailed deer ( Odocoileus virginianus ) to SARS-CoV-2 . J. Virol.

Patz, J.A., Daszak, P., Tabor, G.M., Aguirre, A.A., Pearl, M., Epstein, J., et al. (2004) Unhealthy landscapes: Policy recommendations on land use change and infectious disease emergence. Environ. Health Perspect. 112: 1092-1098.

de Paula, N., Jung, L., Mar, K., Bowen, K., Maglakelidze, M., Fünderich, M., Otieno, M., et al. (2021) A planetary health blind spot : the untapped potential of women to safeguard nature and human resilience in LMICs. Lancet Planet. Heal. 109-110.

Plowright, R.K., Becker, D.J., Crowley, D.E., Washburne, A.D., Huang, T., Nameer, P.O., et al. (2019) Prioritizing surveillance of nipah virus in India. PLoS Negl. Trop. Dis. 13: 1-17.

Plowright, R.K., Parrish, C.R., McCallum, H., Hudson, P.J., Ko, A.I., Graham, A.L., and Lloyd-Smith, J.O. (2017) Pathways to zoonotic spillover. Nat. Rev. Microbiol. 15: 502-510. 
Roche, B., Garchitorena, A., Guégan, J.F., Arnal, A., Roiz, D., Morand, S., et al. (2020) Was the COVID-19 pandemic avoidable? A call for a "solution-oriented" approach in pathogen evolutionary ecology to prevent future outbreaks. Ecol. Lett. 23: 1557-1560.

Rock, M.J. and Degeling, C. (2015) Public health ethics and more-than-human solidarity. Soc. Sci. Med. 129: 61-67.

Ruger, J.P. (2020) Positive Public Health Ethics: Toward Flourishing and Resilient Communities and Individuals. Am. J. Bioeth. 20: 44-54.

Schäfer, A. and Baric, R.S. (2017) Epigenetic landscape during coronavirus infection. Pathogens 6:.

Sironi, M., Hasnain, S.E., Rosenthal, B., Phan, T., Luciani, F., Shaw, M.A., et al. (2020) SARS-CoV-2 and COVID-19: A genetic, epidemiological, and evolutionary perspective. Infect. Genet. Evol. 84: 104384 .

Smith, K.F., Goldberg, M., Rosenthal, S., Carlson, L., Chen, J., Chen, C., and Ramachandran, S. (2014) Global rise in human infectious disease outbreaks. J. R. Soc. Interface 11: 1-6.

Steffen, W., Richardson, K., Rockström, J., Cornell, S.E., Fetzer, I., Bennett, E.M., et al. (2015) Planetary boundaries: Guiding human development on a changing planet. Science (80-. ). 347:

Trottier, J., Darques, R., Ait Mouheb, N., Partiot, E., Bakhache, W., Deffieu, M.S., and Gaudin, R. (2020) Post-lockdown detection of SARS-CoV-2 RNA in the wastewater of Montpellier, France. One Heal. 10: 100157.

Vinke, K., Gabrysch, S., Paoletti, E., Rockström, J., and Schellnhuber, H.J. (2020) Corona and the climate: A comparison of two emergencies. Glob. Sustain. 3: 1-7.

Wang, H., Pipes, L., and Nielsen, R. (2021) Synonymous mutations and the molecular evolution of SARS-CoV-2 origins. Virus Evol. 7: 1-11.

Watts, N., Amann, M., Arnell, N., Ayeb-Karlsson, S., Beagley, J., Belesova, K., et al. (2021) The 2020 report of The Lancet Countdown on health and climate change: responding to converging crises. Lancet 397: 129-170.

WHO (2015) Connecting Global Priorities: Biodiversity and Human Health, a State of Knowledge Review Romanelli, Cristina, Cooper, David, Campbell-Lendrum, Diarmid, Maiero, Marina, Karesh, William B., Hunter, Danny, Golden,C.D. (ed).

WHO (2020) WHO manifesto for a healthy recovery from COVID-19: Prescriptions and Actionables for a Healthy and Green Recovery. World Heal. Organ. 1-5.

Wibmer, C.K., Ayres, F., Hermanus, T., Madzivhandila, M., Kgagudi, P., Lambson, B.E., and Vermeulen, M. (2021) SARS-CoV-2 501Y.V2 escapes neutralization by South African COVID19 donor plasma. bioRxiv.

Woolhouse, M.E.J. and Gowtage-Sequeria, S. (2005) Host range and emerging and reemerging pathogens. Emerg. Infect. Dis. 11: 1842-1847.

Woolhouse, M.E.J., Haydon, D.T., and Antia, R. (2005) Emerging pathogens: The epidemiology and evolution of species jumps. Trends Ecol. Evol. 20: 238-244.

World Health Organization. Food and Agriculture Organization of the United Nations. \& World Organisation for animal health (2019) A Tripartite Guide to Addressing Zoonotic Diseases 
in Countries.

Xie, X., Zou, J., Fontes-Garfias, C.R., Xia, H., Swanson, K.A., Cutler, M., et al. (2021) Neutralization of N501Y mutant SARS-CoV-2 by BNT162b2 vaccine-elicited sera Xuping. bioRxiv 53: 2021.

Zeberg, H. and Pääbo, S. (2020) The major genetic risk factor for severe COVID-19 is inherited from Neandertals Authors. bioRxiv 2020.07.03.186296.

Zhou, P., Yang, X. Lou, Wang, X.G., Hu, B., Zhang, L., Zhang, W., et al. (2020) A pneumonia outbreak associated with a new coronavirus of probable bat origin. Nature 579: 270-273. 